\title{
Small Intestinal Neuroendocrine Tumor
} G1

National Cancer Institute

\section{Source}

National Cancer Institute. Small Intestinal Neuroendocrine Tumor G1. NCI Thesaurus.

Code C4638.

A well differentiated, low grade neuroendocrine tumor (carcinoid tumor) that arises from the small intestine. The mitotic count is less than 2 per $10 \mathrm{HPF}$ and/or the Ki67 index is equal to or less than 2 percent. 